\title{
Viability for nonlinear multi-valued reaction-diffusion systems
}

\author{
Daniela Roşu
}

\begin{abstract}
In this paper we consider a nonlinear evolution reactiondiffusion system governed by multi-valued perturbations of $m$-dissipative operators, generators of nonlinear semigroups of contractions. Let $X$ and $Y$ be real Banach spaces, $\mathcal{K}$ be a nonempty and locally closed subset in $\mathbb{R} \times X \times Y, A: D(A) \subseteq X \rightsquigarrow X, B: D(B) \subseteq Y \rightsquigarrow Y$ two $m$-dissipative operators, $F: \mathcal{K} \rightarrow X$ a continuous function and $G: \mathcal{K} \rightsquigarrow Y$ a nonempty, convex and closed valued, strongly-weakly upper semi-continuous (u.s.c.) multi-function. We prove a necessary and a sufficient condition in order that for each $(\tau, \xi, \eta) \in \mathcal{K}$, the next system
\end{abstract}

$$
\left\{\begin{array}{l}
u^{\prime}(t) \in A u(t)+F(t, u(t), v(t)) \quad t \geq \tau \\
v^{\prime}(t) \in B v(t)+G(t, u(t), v(t)) \quad t \geq \tau \\
u(\tau)=\xi, \quad v(\tau)=\eta
\end{array}\right.
$$

has at least one $C^{0}$-solution $(u, v):[\tau, T] \rightarrow X \times Y$ with $(t, u(t), v(t)) \in \mathcal{K}$ for each $t \in[\tau, T]$.

Mathematics Subject Classification (2000). Primary 34G20, 47J35, 35K57; Secondary 35K45, 35K65.

Keywords. $m$-Dissipative operator, Reaction-diffusion system, Viable set, Tangent set, u.s.c. Multi-function.

\section{Introduction}

In this paper, we prove some viability results referring to a class of nonlinear reaction-diffusion systems governed by multi-valued perturbations of nonlinear $m$-dissipative operators, results announced in Roşu [22]. Let $\left(X,\|\cdot\|_{X}\right)$ and $\left(Y,\|\cdot\|_{Y}\right)$ be real Banach spaces, $A: D(A) \subseteq X \rightsquigarrow X$ and $B: D(B) \subseteq$ $Y \rightsquigarrow Y$ be two $m$-dissipative, possible multi-valued, operators generators of nonlinear semigroups of contractions, $\left\{S_{A}(t): \overline{D(A)} \rightarrow \overline{D(A)} ; t \geq 0\right\}$ and 
$\left\{S_{B}(t): \overline{D(B)} \rightarrow \overline{D(B)} ; t \geq 0\right\}$, respectively. Let $\mathcal{K}$ be a nonempty subset in $\mathbb{R} \times \overline{D(A)} \times \overline{D(B)}$, let $F: \mathcal{K} \rightarrow X$ be a given function and let $G: \mathcal{K} \rightsquigarrow Y$ be a given nonempty valued multi-function. We consider the reaction-diffusion system

$$
\begin{cases}u^{\prime}(t) \in A u(t)+F(t, u(t), v(t)) & t \geq \tau \\ v^{\prime}(t) \in B v(t)+G(t, u(t), v(t)) & t \geq \tau \\ u(\tau)=\xi, \quad v(\tau)=\eta, & \end{cases}
$$

where $(\tau, \xi, \eta) \in \mathcal{K}$.

Definition 1.1. The continuous function $(u, v):[\tau, T] \rightarrow \overline{D(A)} \times \overline{D(B)}$ is a $C^{0}$-solution of the multi-valued Cauchy problem $(1.1)$ on $[\tau, T]$ if $(t, u(t), v(t))$ $\in \mathcal{K}$ for each $t \in[\tau, T]$, the function $f(\cdot)=F(\cdot, u(\cdot), v(\cdot))$ belongs to $L^{1}$ $(\tau, T ; X)$ and there exists $g \in L^{1}(\tau, T ; Y)$ such that $g(s) \in G(s, u(s), v(s))$ a.e. for $s \in[\tau, T]$ and $(u, v)$ is a $C^{0}$-solution on $[\tau, T]$ of the problem

$$
\left\{\begin{array}{l}
u^{\prime}(t) \in A u(t)+f(t) \\
v^{\prime}(t) \in B v(t)+g(t) \\
u(\tau)=\xi, \quad v(\tau)=\eta
\end{array}\right.
$$

Definition 1.2. The set $\mathcal{K}$ is $C^{0}$-viable with respect to $(A+F, B+G)$ if for each $(\tau, \xi, \eta) \in \mathcal{K}$ there exists $T>\tau$ such that the Cauchy problem (1.1) has at least one $C^{0}$-solution on $[\tau, T]$.

The viability problem has been studied by many authors. For the differential inclusion $u^{\prime}(t) \in A u(t)+F(t, u(t))$, when $X$ is finite dimensional, we mention the works of Nagumo [18] (the case in which $A \equiv 0$ and $F$ is singlevalued and continuous) and Bebernes-Schuur [5] (the case in which $A \equiv 0$ and $F$ is u.s.c. multi-function with nonempty, convex, closed and bounded valuescase briefly mentioned by Hukuhara [15], as to admit a similar treatment as the main result of Nagumo [18]). An extension to infinite-dimensional spaces, by imposing an additional hypothesis on $F$ expressed in the terms of the measure of noncompactness, is due to Deimling [14]. For other results, we refer to Papageorgiou [20]. If $X$ is infinite-dimensional, the semilinear case, i.e., the case in which $A$ is the infinitesimal generator of a $C_{0}$-semigroup and $F$ is a continuous function, was studied by Pavel [21]. The fully nonlinear case, i.e., when $A$ is nonlinear and $F$ is a continuous function, has been considered by Vrabie [24]. The basic concepts and results of the Viability Theory, as well as a detailed history of the domain up to 1991, can be found in Aubin [3]. More recent results (in the case in which $F=F(t, u)$ is Carathéodory) can be found in Bothe [6]. The viability problem for semilinear differential inclusions, when $A$ is the infinitesimal generator of a $C_{0}$-semigroup and $F$ is a locally $\beta$-compact multi-function with nonempty, closed and convex values, has been studied by Cârjă-Necula-Vrabie [12]. The case when $A$ generates a differentiable compact semigroup, $K$ is compact and $F$ is u.s.c. with convex compact values, was previously studied by Shi [23]. For the nonlinear evolution inclusions, where $A$ is an $m$-dissipative operator and $F$ is u.s.c. multi-function with nonempty, convex, closed and bounded values, we mention the work of Cârjă-Necula-Vrabie [13]. 
The viability problem for reaction-diffusion systems of the form (1.1) was studied by Burlică-Roşu [8,9], where $A$ and $B$ are linear operators, generators of two $C_{0}$-semigroups and $F, G$ are single-valued, continuous functions such that $A+F$ and/or $B+G$ are locally of $\beta$-compact type. Necula-Vrabie [19] considered the case in which $A$ and $B$ are nonlinear, $m$-dissipative operators, $B$ generates a compact semigroup, $F, G$ are single-valued, continuous functions and $F: \mathbb{R} \times X \times Y \rightarrow X$ is locally Lipschitz with respect to its second argument. The case in which $A$ and $B$ are linear operators, $F$ is as above, and $G$ is a nonempty, convex, weakly compact valued, strongly weakly u.s.c. multi-function, was considered by Burlică [7].

Our paper is divided into five sections as follows: Section 2 is devoted to some basic concepts and results needed in the sequel. In Sect. 3 we present the main results, while Sect. 4 contains the proof of Theorem 3.2. A comparison result for a predator-prey system is given in Sect.5.

\section{Preliminaries}

In this section we collect some notations, definitions and some basic viability results concerning the multi-valued nonlinear problems. For more details we refer to Cârjă [10] and Cârjă-Necula-Vrabie [11].

In that follows, $(X,\|\cdot\|)$ denotes a real Banach space. For $\xi \in X$ and $\rho>0, D(\xi, \rho)$ denotes the closed ball in $X$ of radius $\rho$ centered in $\xi$ and $\operatorname{dist}(E, K)$ denotes the usual distance between the subsets $E$ and $K$.

By an operator or multi-function we mean a mapping (multi-valued map) $A: X \rightarrow 2^{X}$. The domain of $A$ is defined by $D(A)=\{x \in X ; A x \neq \emptyset\}$. In all that follows we shall write $A: D(A) \subseteq X \rightsquigarrow X$ instead $A: X \rightarrow 2^{X}$, to signify that $A x \neq \emptyset$ if and only if $x \in D(A)$.

Let $A: D(A) \subseteq X \rightsquigarrow X$ be an $m$-dissipative operator, $\xi \in \overline{D(A)}$ and $f \in L^{1}(\tau, T ; X)$ and let us consider the Cauchy problem:

$$
\left\{\begin{array}{l}
u^{\prime}(t) \in A u(t)+f(t) \quad t \geq \tau \\
u(\tau)=\xi
\end{array}\right.
$$

Definition 2.1. A $C^{0}$-solution of the problem (2.1) is a function $u \in C([\tau, T] ; X)$ satisfying $u(\tau)=\xi$ and, for each $\tau<c<T$ and $\varepsilon>0$ there exist

(i) $\tau=t_{0}<t_{1}<\cdots<c \leq t_{n}<T, t_{k}-t_{k-1} \leq \varepsilon$ for $k=1,2, \ldots, n$;

(ii) $f_{1}, \ldots, f_{n} \in X$ with $\sum_{k=1}^{n} \int_{t_{k-1}}^{t_{k}}\left\|f(t)-f_{k}\right\| d t \leq \varepsilon$

(iii) $v_{0}, \ldots, v_{n} \in X$ satisfying:

$$
\begin{aligned}
& \frac{v_{k}-v_{k-1}}{t_{k}-t_{k-1}} \in A v_{k}+f_{k} \quad \text { for } \quad k=1,2, \ldots, n \\
& \left\|u(t)-v_{k}\right\| \leq \varepsilon \quad \text { for } \quad t \in\left[t_{k-1}, t_{k}\right), k=1,2, \ldots, n .
\end{aligned}
$$

Theorem 2.1. Let $A: D(A) \subseteq X \rightsquigarrow X$ be an m-dissipative operator. Then, for each $\xi \in \overline{D(A)}$ and $f \in L^{1}(\tau, T ; X)$, there exists a unique $C^{0}$-solution $u:[\tau, T] \rightarrow \overline{D(A)}$ of the problem $(2.1)$. 
See Lakshmikantham-Leela [16], Theorem 3.6.1, p. 116.

We denote by $u(\cdot, \tau, \xi, f):[\tau, T] \rightarrow \overline{D(A)}$ the unique $C^{0}$-solution of $(2.1)$ satisfying $u(\tau, \tau, \xi, f)=\xi$. We recall that, if $\widetilde{u}=u(\cdot, \tau, \xi, f)$ and $\widetilde{v}=u(\cdot, \tau, \eta, g)$, then we have

$$
\|\widetilde{u}(t)-\widetilde{v}(t)\| \leq\|\xi-\eta\|+\int_{\tau}^{t}\|f(s)-g(s)\| d s,
$$

for each $t \in[\tau, T]$. If $A: D(A) \subseteq X \rightsquigarrow X$ is $m$-dissipative we denote by $\{S(t): \overline{D(A)} \rightarrow \overline{D(A)} ; t \geq 0\}$ the nonlinear semigroup of nonexpansive mappings generated by $A$, i.e. $S(t) \xi=u(t, 0, \xi, 0)$ for each $t \geq 0$ and $\xi \in \overline{D(A)}$. For more details referring to evolutions governed by $m$-dissipative operators see Barbu [4], Lakshmikantham-Leela [16] or Vrabie [25].

Let $A: D(A) \subseteq X \rightsquigarrow X$ be an $m$-dissipative operator, $K$ a nonempty subset in $X$ and $F: K \rightsquigarrow X$ a nonempty, closed and convex valued multifunction. We consider the Cauchy problem

$$
\left\{\begin{array}{l}
u^{\prime}(t) \in A u(t)+F(u(t)) \\
u(0)=\xi
\end{array}\right.
$$

Definition 2.2. The function $u:[0, T] \rightarrow K$ is a $C^{0}$-solution of the problem (2.2) if there exists $f \in L^{1}(0, T ; X)$, with $f(s) \in F(u(s))$ a.e. for $s \in[0, T]$, such that $u$ is a $C^{0}$-solution on $[0, T]$ of the problem

$$
\left\{\begin{array}{l}
u^{\prime}(t) \in A u(t)+f(t) \\
u(0)=\xi
\end{array}\right.
$$

in the sense of Definition 2.1.

Definition 2.3. The set $K \subseteq X$ is $C^{0}$-viable with respect to $A+F$ if for each $\xi \in K$, there exists $T>0$ such that the Cauchy problem (2.2) has at least one $C^{0}$-solution $u:[0, T] \rightarrow K$.

In the following we recall the concept of A-tangent set and A-quasitangent set introduced by Cârjă-Necula-Vrabie [11].

Definition 2.4. Let $A: D(A) \subseteq X \leadsto X$ be an $m$-dissipative operator, let $K$ be a subset in $X$ and $\xi \in K$. Let $E$ be a nonempty and bounded subset in $X$. We say that the set $E$ is $A$-tangent to the set $K$ at the point $\xi$ if we have

$$
\liminf _{h \downarrow 0} \frac{1}{h} \operatorname{dist}\left(S_{E}(h) \xi ; K\right)=0,
$$

where

$$
S_{E}(h) \xi=\{u(h, 0, \xi, \eta) ; \eta \in E\} .
$$

Definition 2.5. Let $A: D(A) \subseteq X \leadsto X$ be an $m$-dissipative operator, let $K$ be a subset in $X$ and $\xi \in K$. Let $E$ be a nonempty and bounded subset in $X$. We say that the set $E$ is $A$-quasi-tangent to the set $K$ at the point $\xi$ if we have

$$
\liminf _{h \downarrow 0} \frac{1}{h} \operatorname{dist}\left(S_{\mathcal{E}}(h) \xi ; K\right)=0,
$$

where

$$
S_{\mathcal{E}}(h) \xi=\left\{u(h, 0, \xi, f) ; f \in L_{\mathrm{loc}}^{1}\left(\mathrm{R}_{+} ; X\right), f(s) \in E \quad \text { a.e. for } s \in \mathrm{R}_{+}\right\} .
$$


We denote by $\mathcal{T S}_{K}^{A}(\xi)$ and $2 \mathcal{T} \mathcal{S}_{K}^{A}(\xi)$ the class of all $A$-tangent sets and, respectively, $A$-quasi-tangent sets to $K$ at $\xi \in K$.

Remark 2.1. Identifying constants with locally integrable functions, we conclude that

$$
\mathcal{T} \mathcal{S}_{K}^{A}(\xi) \subseteq Q \mathcal{Q} \mathcal{S}_{K}^{A}(\xi)
$$

We recall that the subset $K \subseteq X$ is locally closed if for each $\xi \in K$ there exists $\rho>0$ such that $D(\xi, \rho) \cap K$ is closed. A subset $C \subseteq X$ is quasiweakly (relatively) compact if, for each $r>0, D(0, r) \cap C$ is weakly (relatively) compact.

We present now a necessary condition for mild viability.

Theorem 2.2. Let $X$ be a Banach space, $A: D(A) \subseteq X \rightsquigarrow X$ an $m$-dissipative operator, $K$ a nonempty subset in $\overline{D(A)}$ and $F: K \rightsquigarrow X$ a nonempty valued multi-function. If $K$ is $C^{0}$-viable with respect to $A+F$ then, for each $\xi \in K$ at which $F$ is u.s.c. and $F(\xi)$ is convex and quasi-weakly compact, we have

$$
F(\xi) \in \mathcal{Q T S}_{K}^{A}(\xi)
$$

For the proof see Theorem 11.1.2, p. 225 in Cârjă-Necula-Vrabie [11].

Definition 2.6. An $m$-dissipative operator $A: D(A) \subseteq X \rightsquigarrow X$ is called of complete continuous type if, for each fixed $(\tau, \xi) \in \mathbb{R} \times \overline{D(A)}$, the graph of the $C^{0}$-solution operator $f \mapsto u(\cdot, \tau, \xi, f)$, is weakly $\times$ strongly sequentially closed in $L^{1}(\tau, T ; X) \times C([\tau, T] ; X)$.

\section{The main results}

We present now the main necessary and sufficient conditions for viability in the case of nonlinear multi-valued reaction-diffusion systems of the form (1.1). Let $\left(X,\|\cdot\|_{X}\right)$ and $\left(Y,\|\cdot\|_{Y}\right)$ be two real Banach spaces, $\mathcal{K}$ a nonempty subset in $\mathbb{R} \times \overline{D(A)} \times \overline{D(B)}, F: \mathcal{K} \rightarrow X$ a continuous function, $G: \mathcal{K} \rightsquigarrow Y$ a given u.s.c. multi-function with nonempty, convex and closed values. We assume that the operators $A: D(A) \subseteq X \rightsquigarrow X$ and $B: D(B) \subseteq Y \rightsquigarrow Y$ are $m$-dissipative and generate nonlinear semigroups of contractions.

Remark 3.1. The system (1.1) can be rewritten as a multi-valued nonlinear autonomous Cauchy problem in a product space. Let $Z=\mathbb{R} \times X \times Y$ endowed with the norm $\|\cdot\|_{Z}$, defined by $\|(t, x, y)\|_{Z}=|t|+\|x\|_{X}+\|y\|_{Y}$, for each $(t, x, y) \in Z$. Let $\mathcal{A}: D(\mathcal{A}) \subseteq Z \rightsquigarrow Z$ be defined by $D(\mathcal{A})=$ $\mathbb{R} \times D(A) \times D(B), \mathcal{A}(z)=(0, A x, B y)$ for each $z=(t, x, y) \in D(\mathcal{A})$ and let $\mathcal{F}: \mathcal{K} \rightsquigarrow Z, \mathcal{F}(z)=(1, F(z), G(z))$ for each $z \in Z$, where $(1, F(z), G(z))=$ $\{(1, F(z), \eta) ; \eta \in G(z)\}$. So, the system (1.1) can be rewritten as 


$$
\left\{\begin{array}{l}
z^{\prime}(s) \in \mathcal{A} z(s)+\mathcal{F}(z(s)) \\
z(0)=\zeta
\end{array}\right.
$$

where $\zeta=(\tau, \xi, \eta)$. In the hypotheses above, $\mathcal{A}$ is $m$-dissipative and therefore it generates a nonlinear semigroup of contractions $\{\mathcal{S}(t): \overline{D(\mathcal{A})} \rightarrow \overline{D(\mathcal{A})}, t \geq 0\}$. For $f \in L^{1}(\tau, T ; X), g \in L^{1}(\tau, T ; Y)$ and $(\xi, \eta) \in \overline{D(A)} \times \overline{D(B)}$, we denote by $(u(\cdot, \tau, \xi, \eta, f), v(\cdot, \tau, \xi, \eta, g))$ the unique $C^{0}$-solution on $[\tau, T]$ of $(1.2)$. Let us remark that the function $z=(t, x, y)$ is a $C^{0}$-solution of (3.4) if and only if

$$
\left\{\begin{array}{l}
t(s)=\tau+s \\
x(s)=u(\tau+s, \tau, \xi, \eta, f(\tau+\cdot)) \\
y(s)=v(\tau+s, \tau, \xi, \eta, g(\tau+\cdot))
\end{array}\right.
$$

for each $s \in[0, T-\tau]$ where $f, g$ are the functions in Definition 1.1.

Remark 3.2. We also remark that $\mathcal{K}$ is $C^{0}$-viable with respect to $(A+F, B+G)$ in sense of Definition 1.2 if and only if $\mathcal{K}$ is $C^{0}$-viable with respect to $\mathcal{A}+\mathcal{F}$ in sense of Definition 2.3.

From Theorem 2.2 and Remarks 3.1 and 3.2, we deduce the necessary condition in order that $\mathcal{K}$ be $C^{0}$-viable with respect to $(A+F, B+G)$.

Theorem 3.1. Let $X$ and $Y$ be Banach spaces, let $A: D(A) \subseteq X \rightsquigarrow X$ and $B: D(B) \subseteq Y \rightsquigarrow Y$ be $m$-dissipative operators, $\mathcal{K}$ a nonempty subset in $\mathbb{R} \times \overline{D(A)} \times \overline{D(B)}, F: \mathcal{K} \rightarrow X$ a continuous function and $G: \mathcal{K} \rightsquigarrow Y a$ nonempty, convex and quasi-weakly compact valued, u.s.c. multi-function. If $\mathcal{K}$ is $C^{0}$-viable with respect to $(A+F, B+G)$ then, for each $(\tau, \xi, \eta) \in \mathcal{K}$ we have:

$$
(1, F(\tau, \xi, \eta), G(\tau, \xi, \eta)) \in Q \mathcal{Q} \mathcal{S}_{\mathcal{K}}^{\mathcal{A}}(\tau, \xi, \eta)
$$

The main sufficient condition of $C^{0}$-viability is:

Theorem 3.2. Let $X$ and $Y$ be Banach spaces, $A: D(A) \subseteq X \rightsquigarrow X$ and $B: D(B) \subseteq Y \rightsquigarrow Y$ be $m$-dissipative operators. Assume that the operator $B$ is of complete continuous type which generates a compact semigroup of contractions. Let $\mathcal{K}$ be a nonempty and locally closed subset in $\mathbb{R} \times \overline{D(A)} \times \overline{D(B)}$. Let $F: \mathbb{R} \times X \times Y \rightarrow X$ be continuous and globally Lipschitz with respect to its second argument, $u$, i.e. there exists $L>0$ such that

$$
\|F(t, u, v)-F(t, \widetilde{u}, v)\|_{X} \leq L\|u-\widetilde{u}\|_{X}
$$

for each $(t, u, v),(t, \widetilde{u}, v) \in \mathbb{R} \times X \times Y$. Let $G: \mathcal{K} \rightsquigarrow Y$ be a nonempty, convex and weakly compact valued, strongly-weakly u.s.c. and locally bounded multifunction. Then a sufficient condition in order that $\mathcal{K}$ be $C^{0}$-viable with respect to $(A+F, B+G)$ is that, for each $(\tau, \xi, \eta) \in \mathcal{K}$ the tangency condition (3.2) be satisfied.

From Theorem 3.2 and Remark 2.1, we deduce:

Corollary 3.1. Let $X$ and $Y$ be Banach spaces, $A: D(A) \subseteq X \rightsquigarrow X$ and $B: D(B) \subseteq Y \rightsquigarrow Y$ be $m$-dissipative operators. Assume that the operator $B$ is of complete continuous type which generates a compact semigroup of contractions. Let $\mathcal{K}$ be a nonempty and locally closed subset in $\mathbb{R} \times \overline{D(A)} \times \overline{D(B)}$. 
Let $F: \mathbb{R} \times X \times Y \rightarrow X$ be continuous and globally Lipschitz with respect to its second argument, $u$, i.e., there exists $L>0$ such that

$$
\|F(t, u, v)-F(t, \widetilde{u}, v)\|_{X} \leq L\|u-\widetilde{u}\|_{X}
$$

for each $(t, u, v),(t, \widetilde{u}, v) \in \mathbb{R} \times X \times Y$. Let $G: \mathcal{K} \rightsquigarrow Y$ be a nonempty, convex and weakly compact valued, strongly-weakly u.s.c. and locally bounded multifunction. Then a sufficient condition in order that $\mathcal{K}$ be $C^{0}$-viable with respect to $(A+F, B+G)$ is that, for each $(\tau, \xi, \eta) \in \mathcal{K}$ the tangency condition

$$
(1, F(\tau, \xi, \eta), G(\tau, \xi, \eta)) \in \mathcal{T S}_{\mathcal{K}}^{\mathcal{A}}(\tau, \xi, \eta)
$$

be satisfied.

\section{Proof of Theorem 3.2}

Since a direct proof of Corollary 3.1 is almost identical and thus by no means simpler than the one of Theorem 3.2, we will prove Theorem 3.2.

We start this section with a few words about the proof of our main result, i.e., Theorem 3.2.

As one may easily observe, if we fix $v$ in the first equation, we get a differential equation which can be analyzed by means of Theorem 10.5.2, p. 210 in Cârjă-Necula-Vrabie [7]. Further, if we fix $u$ in the second one, we get a differential inclusion which can be handled by Theorem 11.6.4, p. 236, loc. cit. Nevertheless, we emphasize that, as far as we can see, it is impossible to prove our main result by successive applications of the two results mentioned above. In fact, in this context, the challenging point is to show that the double-sequence of $\varepsilon$-approximate $C^{0}$-solutions has at least one convergent subsequence. To summarize, we start with Lemma 11.3.1, p. 227 in Cârjă-Necula-Vrabie [7] and we obtain a double-sequence of $\varepsilon$-approximate $C^{0}$-solutions. We continue with an appropriate interplay between the Lipschitz condition of $F$ and the compactness of the semigroup generated by $B$ in order to get a uniformly convergent double-subsequence. Finally, we get the conclusion by passing to the limit in the corresponding $\varepsilon$-approximate equations.

Proof. From Remark 3.2, it suffices to show that the set $\mathcal{K}$ is $C^{0}$-viable with respect to $\mathcal{A}+\mathcal{F}$. Let $\zeta=(\tau, \xi, \eta) \in \mathcal{K}$ be arbitrary. We will prove that the problem

$$
\left\{\begin{array}{l}
z^{\prime}(s) \in \mathcal{A} z(s)+\mathcal{F}(z(s)) \\
z(0)=\zeta
\end{array}\right.
$$

has a $C^{0}$-solution. Let $\left(\varepsilon_{n}\right)_{n} \downarrow 0$ be a sequence in $(0,1)$. The tangency condition (3.2) is equivalent to

$$
\mathcal{F}(\zeta) \in Q \mathcal{T} \mathcal{S}_{\mathcal{K}}^{\mathcal{A}}(\zeta)
$$

for each $\zeta \in \mathcal{K}$. Since $\mathcal{F}$ is locally bounded, from Lemma 11.3.1, p. 227 in Cârjă-Necula-Vrabie [11] we conclude that there exist $\rho>0, T>0, M>0$ 
such that $D_{Z}(\zeta, \rho) \cap \mathcal{K}$ is closed, $\|w\|_{Z} \leq M$ for each $w \in \mathcal{F}(z)$ and $z \in$ $D_{Z}(\zeta, \rho) \cap \mathcal{K}$ and

$$
\sup _{t \in[0, T]}\|\mathcal{S}(t) \zeta-\zeta\|_{Z}+T(M+1) \leq \rho .
$$

Moreover there exists $\left(\alpha_{n}, \widetilde{f}_{n}, w_{n}\right)_{n}$ an $\varepsilon_{n}$-approximate $C^{0}$-solutions of (4.1). This means that $\alpha_{n}:[0, T] \rightarrow[0, T]$ is nondecreasing, $\widetilde{f}_{n} \in L^{\infty}(0, T ; Z)$ and $w_{n}:[0, T] \rightarrow Z$ is continuous and the next conditions are satisfied.

(i) $s-\varepsilon_{n} \leq \alpha_{n}(s) \leq s$ for each $s \in[0, T]$;

(ii) $w_{n}\left(\alpha_{n}(s)\right) \in D_{Z}(\zeta, \rho) \cap \mathcal{K}$ for each $s \in[0, T], w_{n}(T) \in D_{Z}(\zeta, \rho) \cap \mathcal{K}$ and $w_{n}([0, T])$ is precompact;

(iii) $\tilde{f}_{n}(s) \in \mathcal{F}\left(w_{n}\left(\alpha_{n}(s)\right)\right)$ a.e. for $s \in[0, T]$;

(iv) $\left\|\widetilde{f}_{n}(s)\right\|_{Z} \leq M$ a.e. for $s \in[0, T]$;

(v) $w_{n}(0)=\zeta$ and $\| w_{n}(s)-u\left(s, \alpha_{n}(r), w_{n}\left(\alpha_{n}(r), \tilde{f}_{n}\right) \|_{Z} \leq\left(s-\alpha_{n}(r)\right) \varepsilon_{n}\right.$ for all $0 \leq r \leq s \leq T$;

(vi) $\left\|w_{n}(s)-w_{n}\left(\alpha_{n}(s)\right)\right\|_{Z} \leq \varepsilon_{n}$ for each $s \in[0, T]$.

We note $\tilde{f}_{n}:=\left(\theta_{n}, f_{n}, g_{n}\right)$ and $w_{n}:=\left(\sigma_{n}, x_{n}, y_{n}\right)$. By $(\mathrm{v})$, for $r=0$, we have

$$
\left\|w_{n}(s)-u\left(s, 0, \zeta, \tilde{f}_{n}\right)\right\|_{Z} \leq s \varepsilon_{n}
$$

for each $s \in[0, T]$, where $u\left(\cdot, 0, \zeta, \widetilde{f}_{n}\right)$ is the $C^{0}$-solution for the problem:

$$
\left\{\begin{array}{l}
z^{\prime}(s) \in \mathcal{A} z(s)+\widetilde{f}_{n}(s) \\
z(0)=\zeta .
\end{array}\right.
$$

This means that $u\left(\cdot, 0, \zeta, \widetilde{f}_{n}\right)=\left(\cdot+\tau, x\left(\cdot, 0, \xi, \eta, f_{n}\right), y\left(\cdot, 0, \xi, \eta, g_{n}\right)\right)$ where $(x(\cdot), y(\cdot))=\left(x\left(\cdot, 0, \xi, \eta, f_{n}\right), y\left(\cdot, 0, \xi, \eta, g_{n}\right)\right)$ is the unique $C^{0}$-solution for the problem:

$$
\left\{\begin{array}{l}
x^{\prime}(s) \in A x(s)+f_{n}(s) \\
y^{\prime}(s) \in B y(s)+g_{n}(s) \\
x(0)=\xi, y(0)=\eta
\end{array}\right.
$$

We will prove that, on a subsequence at least, $\left(u\left(\cdot, 0, \zeta, \widetilde{f}_{n}\right)\right)_{n}$ is uniformly convergent on $[0, T]$ to a function $\widetilde{z}$ which is a $C^{0}$-solution of (4.1). First of all, from (4.3), we deduce that $\lim _{n} \sigma_{n}(s)=s+\tau$ uniformly for $s \in[0, T]$. From (vi), we obtain that

$$
\lim _{n} \sigma_{n}\left(\alpha_{n}(s)\right)=s+\tau
$$

uniformly for $s \in[0, T]$.

By (iv), $\left\{g_{n} ; n=1,2, \ldots\right\}$ is uniformly bounded, and hence it is uniformly integrable in $L^{1}(0, T ; Y)$. Since $B$ is $m$-dissipative and generates a compact semigroup, thanks to Theorem 2.3.3 (Baras), p. 47 in Vrabie [25], there exists $\widetilde{y} \in C([0, T] ; Y)$ such that, on a subsequence at least,

$$
\lim _{n} y\left(s, 0, \xi, \eta, g_{n}\right)=\widetilde{y}(s)
$$


uniformly for $s \in[0, T]$. By (4.7) and (4.3), we have $\lim _{n} y_{n}(s)=\widetilde{y}(s)$ uniformly for $s \in[0, T]$. From (vi), we conclude that

$$
\lim _{n} y_{n}\left(\alpha_{n}(s)\right)=\widetilde{y}(s)
$$

uniformly for $s \in[0, T]$.

Next, let us consider the Cauchy problem

$$
\left\{\begin{array}{l}
x^{\prime}(s) \in A x(s)+F(\tau+s, x(s), \widetilde{y}(s)) \\
x(0)=\xi
\end{array}\right.
$$

Since $A$ is $m$-dissipative and the function $f_{0}:[0, T] \times X \rightarrow X$, defined by $f_{0}(s, x)=F(\tau+s, x, \widetilde{y}(s))$ for each $(s, x) \in[0, T] \times X$, is globally Lipschitz with respect to $x \in X$, there exits $T_{0} \in(0, T]$ such that the problem (4.9) has an unique $C^{0}$-solution $\widetilde{x}:\left[0, T_{0}\right] \rightarrow X$. From (3.4), we deduce that $f_{0}$ is positively sublinear. See Definition 3.6.1, p. 78 in Cârjă-Necula-Vrabie [11]. Taking into account of $(4.2)$, we obtain that $T_{0}=T$. Thus there exists a $C^{0}$-solution of $(4.9), \widetilde{x} \in C([0, T] ; X)$. Let us prove now that $\lim _{n} x\left(s, 0, \xi, \eta, f_{n}\right)=\widetilde{x}(s)$ uniformly for $s \in[0, T]$.

Since $f_{n}(s)=F\left(w_{n}\left(\alpha_{n}(s)\right)\right)$ a.e. for $s \in[0, T]$, we have

$$
\begin{gathered}
\left\|x\left(s, 0, \xi, \eta, f_{n}\right)-\widetilde{x}(s)\right\|_{X} \leq \int_{0}^{s} \| F\left(\sigma_{n}\left(\alpha_{n}(r)\right), x_{n}\left(\alpha_{n}(r)\right), y_{n}\left(\alpha_{n}(r)\right)\right) \\
-F(\tau+r, \widetilde{x}(r), \widetilde{y}(r)) \|_{X} d r
\end{gathered}
$$

for $n=1,2, \ldots$ and each $s \in[0, T]$.

From (3.4), we deduce

$$
\begin{aligned}
\| F( & \left.\sigma_{n}\left(\alpha_{n}(r)\right), x_{n}\left(\alpha_{n}(r)\right), y_{n}\left(\alpha_{n}(r)\right)\right)-F(\tau+r, \widetilde{x}(r), \widetilde{y}(r)) \|_{X} \\
\leq & \left\|F\left(\sigma_{n}\left(\alpha_{n}(r)\right), x_{n}\left(\alpha_{n}(r)\right), y_{n}\left(\alpha_{n}(r)\right)\right)-F\left(\sigma_{n}\left(\alpha_{n}(r)\right), \widetilde{x}(r), y_{n}\left(\alpha_{n}(r)\right)\right)\right\|_{X} \\
& +\left\|F\left(\sigma_{n}\left(\alpha_{n}(r)\right), \widetilde{x}(r), y_{n}\left(\alpha_{n}(r)\right)\right)-F(\tau+r, \widetilde{x}(r), \widetilde{y}(r))\right\|_{X} \\
\leq & L\left\|x_{n}\left(\alpha_{n}(r)\right)-\widetilde{x}(r)\right\|_{X}+\| F\left(\sigma_{n}\left(\alpha_{n}(r)\right), \widetilde{x}(r), y_{n}\left(\alpha_{n}(r)\right)\right) \\
& -F(\tau+r, \widetilde{x}(r), \widetilde{y}(r)) \|_{X}
\end{aligned}
$$

for $n=1,2, \ldots$ and each $r \in[0, T]$.

By (vi) and (4.3), we have

$$
\begin{aligned}
& \left\|x_{n}\left(\alpha_{n}(r)\right)-\widetilde{x}(r)\right\|_{X} \leq\left\|x_{n}\left(\alpha_{n}(r)\right)-x_{n}(r)\right\|_{X} \\
& \quad+\left\|x_{n}(r)-x\left(r, 0, \xi, \eta, f_{n}\right)\right\|_{X}+\left\|x\left(r, 0, \xi, \eta, f_{n}\right)-\widetilde{x}(r)\right\|_{X} \\
& \leq(1+T) \varepsilon_{n}+\left\|x\left(r, 0, \xi, \eta, f_{n}\right)-\widetilde{x}(r)\right\|_{X}
\end{aligned}
$$

for $n=1,2, \ldots$ and each $r \in[0, T]$.

On the other hand, by (4.6), (4.8) and the continuity of $F$ on $\mathbb{R} \times X \times Y$, we deduce that there exists $\gamma_{n} \downarrow 0$ such that

$$
\left\|F\left(\sigma_{n}\left(\alpha_{n}(r)\right), \widetilde{x}(r), y_{n}\left(\alpha_{n}(r)\right)\right)-F(\tau+r, \widetilde{x}(r), \widetilde{y}(r))\right\|_{X} \leq \gamma_{n}
$$

for $n=1,2, \ldots$ and each $r \in[0, T]$. 
Thus, from (4.10), (4.11), (4.12) and (4.13), we get

$$
\begin{aligned}
& \left\|x\left(s, 0, \xi, \eta, f_{n}\right)-\widetilde{x}(s)\right\|_{X} \leq L T(1+T) \varepsilon_{n}+T \gamma_{n} \\
& \quad+L \int_{0}^{s}\left\|x\left(r, 0, \xi, \eta, f_{n}\right)-\widetilde{x}(r)\right\|_{X} d r
\end{aligned}
$$

for $n=1,2, \ldots$ and each $s \in[0, T]$. By Gronwall's Lemma, we conclude

$$
\left\|x\left(s, 0, \xi, \eta, f_{n}\right)-\widetilde{x}(s)\right\|_{X} \leq\left(L T(1+T) \varepsilon_{n}+T \gamma_{n}\right) e^{L T}
$$

for each $n=1,2, \ldots$ and each $s \in[0, T]$. Consequently $\lim _{n} x\left(s, 0, \xi, \eta, f_{n}\right)=$ $\widetilde{x}(s)$ uniformly for $s \in[0, T]$ and, by (4.3) and (vi), we deduce

$$
\lim _{n} x_{n}(s)=\lim _{n} x_{n}\left(\alpha_{n}(s)\right)=\widetilde{x}(s)
$$

uniformly for $s \in[0, T]$.

So, from (4.6), (4.8) and (4.14), we have

$$
\lim _{n} w_{n}\left(\alpha_{n}(s)\right)=(s+\tau, \widetilde{x}(s), \widetilde{y}(s))
$$

uniformly for $s \in[0, T]$. From (ii), we deduce that $(s+\tau, \widetilde{x}(s), \widetilde{y}(s)) \in$ $D_{Z}(\zeta, \rho) \cap \mathcal{K}$ for each $s \in[0, T]$.

Since $\left\{w_{n}\left(\alpha_{n}(s)\right) ; n=1,2, \ldots, s \in[0, T]\right\}$ is relatively compact and $\mathcal{F}$ is strongly weakly u.s.c. with nonempty and weakly compact values, from Lemma 2.6.1, p. 47 in Cârjă-Necula-Vrabie [11], we conclude that the set

$$
C=\overline{\operatorname{conv}} \bigcup_{n=1}^{\infty} \mathcal{F}\left(w_{n}\left(\alpha_{n}([0, T])\right)\right)
$$

is weakly compact in Z. Since $\tilde{f}_{n} \in L^{\infty}(0, T ; Z)$ and $\tilde{f}_{n}(s) \in C$ a.e. for $s \in[0, T]$, from Corollary 1.3.1, p. 10 in Cârjă-Necula-Vrabie [11], we deduce that the set $\left\{\tilde{f}_{n}, n=1,2, \ldots\right\}$ is weakly relatively compact in $L^{1}(0, T ; Z)$. Consequently there exists $\tilde{f} \in L^{1}(0, T ; Z)$ such that, on a subsequence at least, $\lim _{n} \widetilde{f}_{n}=\widetilde{f}$, weakly in $L^{1}(0, T ; Z)$. Let us denote $\widetilde{f}=\left(f^{\mathbb{R}}, f, g\right)$. So we have $\lim _{n} \theta_{n}=f^{\mathbb{R}}$ weakly in $L^{1}(0, T ; \mathbb{R}), \lim _{n} f_{n}=f$ weakly in $L^{1}(0, T ; X)$ and $\lim _{n} g_{n}=g$ weakly in $L^{1}(0, T ; Y)$. From (iii) we have $\theta_{n}(s)=1$ a.e. for $s \in[0, T]$, so

$$
f^{\mathbb{R}}(s)=1 .
$$

Since $f_{n}(s)=F\left(w_{n}\left(\alpha_{n}(s)\right)\right)$ a.e. for $s \in[0, T]$, from (4.15) and the continuity of $F$, we deduce that

$$
f(s)=F(s+\tau, \widetilde{x}(s), \widetilde{y}(s)),
$$

a.e. for $s \in[0, T]$. We notice that, in fact, $\lim _{n} f_{n}=f$ strongly in $L^{1}(0, T ; X)$.

On the other hand, $\lim _{n} g_{n}=g$ weakly in $L^{1}(0, T ; Y)$. As $B$ is of complete continuous type, from (4.7), we conclude that $\widetilde{y}(s)=y(s, 0, \xi, \eta, g)$. Since $G$ is strongly-weakly u.s.c. with nonempty, convex and closed values and $g_{n}(s) \in$ $G\left(w_{n}\left(\alpha_{n}(s)\right)\right)$ a.e. for $s \in[0, T]$, from (4.15) and Lemma 2.6.2, p. 47 in CârjăNecula-Vrabie [11], we conclude that $g(s) \in G(\tau+s, \widetilde{x}(s), \widetilde{y}(s))$ a.e. for $s \in$ $[0, T]$. Thus $\widetilde{z}(s)=(\tau+s, \widetilde{x}(s), \widetilde{y}(s)), s \in[0, T]$, is a $C^{0}$-solution of the problem (4.1), and this completes the proof. 


\section{An example}

Let $\Omega \subseteq \mathbb{R}^{n}, n=1,2, \ldots$ be a bounded domain with $C^{2}$ boundary $\Gamma$. We consider the nonlinear multi-valued reaction-diffusion system

$$
\begin{cases}u_{t}=\Delta \varphi(u)+f(u, v) & (t, x) \in Q_{\tau, T} \\ v_{t} \in \Delta \psi(v)+\left[g_{1}(u, v), g_{2}(u, v)\right] & (t, x) \in Q_{\tau, T} \\ \varphi(u(t, x))=\psi(v(t, x))=0 & (t, x) \in \Sigma_{\tau, T} \\ u(\tau, x)=\xi(x), \quad v(\tau, x)=\eta(x) & x \in \Omega,\end{cases}
$$

where $0 \leq \tau<T \leq \infty, Q_{\tau, T}=(\tau, T) \times \Omega, \Sigma_{\tau, T}=(\tau, T) \times \Gamma, \Delta$ is the usual Laplace operator, $\varphi: \mathbb{R} \rightarrow \mathbb{R}$ and $\psi: \mathbb{R} \rightarrow \mathbb{R}$ are two continuous and nondecreasing functions with $\varphi(0)=\psi(0)=0, f: \mathbb{R} \times \mathbb{R} \rightarrow \mathbb{R}_{+}, g_{i}: \mathbb{R} \times \mathbb{R} \rightarrow \mathbb{R}$ for $i=1,2$ are given functions. We assume that $\xi, \eta \in L^{1}(\Omega)$ and $f$ is continuous function, $g_{1}$ is l.s.c., $g_{2}$ is u.s.c. function and

$$
g_{1}(u, v) \leq g_{2}(u, v)
$$

for each $(u, v) \in \mathbb{R} \times \mathbb{R}$.

Remark 5.1. According to Murray [17, Section 3.3], one possible choice of $f$ and $g_{1}, g_{2}$ in the case of a realistic predator-prey system (without diffusion) is

$$
f(u, v)=-a u^{+}+\frac{\alpha v^{+}}{1+\beta v^{+}} u^{+}
$$

where $a, \alpha, \beta>0$ and $w^{+}=\max \{w, 0\}$, while

$$
g_{1}(u, v)=g_{2}(u, v)=g(u, v)=r\left(1-\frac{v^{+}}{K}\right) v^{+}-\frac{p v^{+}}{1+q v^{+}} u^{+},
$$

with $r, p, q, K>0$ where $u^{+}=u^{+}(t, x)$ is the density of predators, while $v^{+}=v^{+}(t, x)$ is the density of prey at time $t \geq 0$ and point $x \in \Omega$. For a model taking into consideration the diffusion process but with the very same interaction functions $f$ and $g$, see Ainseba-Aniţa [1]. One limiting case, motivated by practical examples, corresponds to $K=+\infty$ and to the multi-valued choice of $g$ as

$$
g(u, v)= \begin{cases}0, & \text { for } v<0, u \in \mathbb{R} \\ -\left[0, \frac{p}{q}\right] u^{+}, & \text {for } v=0, u \in \mathbb{R} \\ r v-\frac{p}{q} u^{+}, & \text {for } v>0, u \in \mathbb{R} .\end{cases}
$$

Here, we have

$$
\begin{aligned}
& g_{1}(u, v)= \begin{cases}0, & \text { for } v<0, u \in \mathbb{R} \\
-\frac{p}{q} u^{+}, & \text {for } v=0, u \in \mathbb{R} \\
r v-\frac{p}{q} u^{+}, & \text {for } v>0, u \in \mathbb{R}\end{cases} \\
& g_{2}(u, v)= \begin{cases}0, & \text { for } v \leq 0, u \in \mathbb{R} \\
r v-\frac{p}{q} u^{+}, & \text {for } v>0, u \in \mathbb{R} .\end{cases}
\end{aligned}
$$

For other possible choices of $f$ and $g$ see Aris [2] or Murray [17]. 
Let $\tilde{f}: \mathbb{R} \times \mathbb{R} \rightarrow \mathbb{R}_{+}$and $\widetilde{g}: \mathbb{R} \times \mathbb{R} \rightarrow \mathbb{R}$ be two continuous functions such that

$$
\left\{\begin{array}{l}
f(u, v) \leq \widetilde{f}(u, v) \\
g_{2}(u, v) \geq \widetilde{g}(u, v)
\end{array}\right.
$$

for each $(u, v) \in \mathbb{R} \times \mathbb{R}$. Let us also consider the comparison system

$$
\begin{cases}u_{t}=\Delta \varphi(u)+\widetilde{f}(u, v) & (t, x) \in Q_{0, \infty} \\ v_{t}=\Delta \psi(v)+\widetilde{g}(u, v) & (t, x) \in Q_{0, \infty} \\ \varphi(u(t, x))=\psi(v(t, x))=0 & (t, x) \in \Sigma_{0, \infty} \\ u(0, x)=u_{0}(x), \quad v(0, x)=v_{0}(x) & x \in \Omega,\end{cases}
$$

where $u_{0}, v_{0} \in L^{\infty}(\Omega), u_{0}(x) \geq 0, v_{0}(x) \geq 0$ a.e. for $x \in \Omega$. Let $(\widetilde{u}, \widetilde{v})$ : $\mathbb{R}_{+} \times \bar{\Omega} \rightarrow \mathbb{R}_{+} \times \mathbb{R}_{+}$be a $C^{0}$-solution of (5.4)

We want to prove some sufficient conditions in order that, for each $(\xi, \eta) \in$ $L^{\infty}(\Omega) \times L^{\infty}(\Omega)$ with

$$
\left\{\begin{array}{l}
0 \leq \xi(x) \leq \widetilde{u}(\tau, x) \\
\eta(x) \geq \widetilde{v}(\tau, x)
\end{array}\right.
$$

a.e. for $x \in \Omega$, the system (5.1) has at least one $C^{0}$-solution $(u, v):[\tau, \infty) \times \Omega \rightarrow$ $\mathbb{R}_{+} \times \mathbb{R}_{+}$, such that, for each $t \in[\tau, \infty)$ and a.a. for $x \in \Omega$, we have

$$
\left\{\begin{array}{l}
0 \leq u(t, x) \leq \widetilde{u}(t, x) \\
v(t, x) \geq \widetilde{v}(t, x)
\end{array}\right.
$$

Let $\mathcal{K} \subseteq \mathbb{R}_{+} \times L^{1}(\Omega) \times L^{1}(\Omega)$ be defined by

$$
\begin{aligned}
\mathcal{K}=\left\{(t, u, v) \in \mathbb{R}_{+} \times L^{\infty}(\Omega) \times L^{\infty}(\Omega) ;(u, v) \text { satisfies }(5.8) \text { below }\right\} \\
\left\{\begin{array}{l}
0 \leq u(x) \leq \widetilde{u}(t, x) \\
v(x) \geq \widetilde{v}(t, x)
\end{array}\right.
\end{aligned}
$$

a.e. for $x \in \Omega$.

Theorem 5.1. Let $\Omega \subseteq \mathbb{R}^{n}, n=1,2, \ldots$, be a bounded domain with $C^{2}$ boundary $\Gamma$. Assume that the next hypotheses are satisfied:

$\left(\mathrm{H}_{1}\right) \varphi: \mathbb{R} \rightarrow \mathbb{R}$ and $\psi: \mathbb{R} \rightarrow \mathbb{R}$ are continuous and nondecreasing functions with $\varphi(0)=\psi(0)=0$;

$\left(\mathrm{H}_{2}\right) \quad \psi \in C^{1}(\mathbb{R} \backslash\{0\} ; \mathbb{R})$ and there exist $c>0$ and $p>\max \{(n-2) / 2,0\}$ such that

$$
\psi^{\prime}(r) \geq c|r|^{p-1}
$$

for each $r \in \mathbb{R} \backslash\{0\}$;

$\left(\mathrm{H}_{3}\right) f: \mathbb{R} \times \mathbb{R} \rightarrow \mathbb{R}_{+}$is continuous on $\mathbb{R} \times \mathbb{R}$ and globally Lipschitz with respect to its first argument, i.e., there exists $L>0$ such that

$$
|f(u, v)-f(\widetilde{u}, v)| \leq L|u-\widetilde{u}|
$$

for each $(u, v),(\widetilde{u}, v) \in \mathbb{R} \times \mathbb{R}$; 
$\left(\mathrm{H}_{4}\right) g_{1}: \mathbb{R} \times \mathbb{R} \rightarrow \mathbb{R}$ is l.s.c., $g_{2}: \mathbb{R} \times \mathbb{R} \rightarrow \mathbb{R}$ is u.s.c.; they satisfy (5.2) and both have linear increasing, i.e., there exist the constants $c_{i j} \geq 0$, $i, j=1, \ldots, 3$ such that

$$
\left|g_{i}(u, v)\right| \leq c_{i 1}|u|+c_{i 2}|v|+c_{i 3},
$$

for each $(u, v) \in \mathbb{R} \times \mathbb{R}, i=1,2$;

$\left(\mathrm{H}_{5}\right) \widetilde{f}: \mathbb{R} \times \mathbb{R} \rightarrow \mathbb{R}_{+}, \widetilde{g}: \mathbb{R} \times \mathbb{R} \rightarrow \mathbb{R}$ are continuous, satisfy (5.3) and there exist the constants $c_{i} \geq 0, i=1, \ldots, 5$ such that

$$
|\widetilde{f}(u, v)| \leq c_{1}|u|+c_{2},
$$

and

$$
|\widetilde{g}(u, v)| \leq c_{3}|u|+c_{4}|v|+c_{5}
$$

for each $(u, v) \in \mathbb{R} \times \mathbb{R}$

$\left(\mathrm{H}_{6}\right)$ for each $\left(u_{0}, v_{0}\right) \in \mathbb{R} \times \mathbb{R}, u \mapsto \widetilde{f}\left(u, v_{0}\right)$ and $v \mapsto \widetilde{g}\left(u_{0}, v\right)$ are nondecreasing, and the applications $u \mapsto \widetilde{g}\left(u, v_{0}\right)$ and $v \mapsto \widetilde{f}\left(u_{0}, v\right)$ are nonincreasing.

Let $\left(u_{0}, v_{0}\right) \in L^{\infty}(\Omega) \times L^{\infty}(\Omega)$ with $u_{0}(x) \geq 0$ and $v_{0}(x) \geq 0$ a.e. for $x \in \Omega$ and let $(\widetilde{u}, \widetilde{v}): \mathbb{R}_{+} \times \bar{\Omega} \rightarrow \mathbb{R}_{+} \times \mathbb{R}_{+}$be a global $C^{0}$-solution of (5.4). Let $\mathcal{K}$ be defined by (5.7). Then, for each $(\tau, \xi, \eta) \in \mathcal{K}$, the problem (5.1) has at least one global $C^{0}$-solution $(u, v):[\tau, \infty) \rightarrow L^{\infty}(\Omega) \times L^{\infty}(\Omega)$ satisfying $(t, u(t), v(t)) \in \mathcal{K}$ for each $t \in[\tau, \infty)$.

Remark 5.2. In the case of $f$ and $g$, defined as in Remark 5.1, we can choose

$$
\widetilde{f}(u, v)=\left(-a+\frac{\alpha}{\beta}\right) u^{+} \text {and } \widetilde{g}(u, v)=r v^{+}-\frac{p}{q} u^{+}
$$

for each $(u, v) \in \mathbb{R} \times \mathbb{R}$, which satisfy all the hypotheses of Theorem 5.1, with $a<\frac{\alpha}{\beta}$. We also notice that, although $g_{1}$ has nothing to do with the comparison system, we do not replace $g_{1}$ by a constant function, because this change is not consistent with the mathematical model considered.

We can now proceed to the proof of Theorem 5.1.

Proof. We will prove that $\mathcal{K}$ is $C^{0}$-viable with respect to $\left(\Delta \varphi+f, \Delta \psi+\left[g_{1}, g_{2}\right]\right)$ and each $C^{0}$-solution $(u, v)$ with $(t, u(t), v(t)) \in \mathcal{K}$ for each $t \in[\tau, T)$, can be extended to a global one obeying the same conditions.

Let us denote by $X=L^{1}(\Omega)$ and let us define the operators: $A: D(A) \subseteq$ $X \rightarrow X$ by

$$
\left\{\begin{array}{l}
D(A):=\left\{u \in L^{1}(\Omega) ; \varphi(u) \in W_{0}^{1,1}(\Omega), \Delta \varphi(u) \in L^{1}(\Omega)\right\} \\
A u:=\Delta \varphi(u) \text { for } u \in D(A),
\end{array}\right.
$$

and, respectively, $B: D(B) \subseteq X \rightarrow X$ by

$$
\left\{\begin{array}{l}
D(B):=\left\{v \in L^{1}(\Omega) ; \psi(v) \in W_{0}^{1,1}(\Omega), \Delta \psi(v) \in L^{1}(\Omega)\right\} \\
B v:=\Delta \psi(v) \text { for } v \in D(B) .
\end{array}\right.
$$

It is known that $A$ and $B$ are $m$-dissipative operators, $\overline{D(A)}=\overline{D(B)}=$ $L^{1}(\Omega)$ and $B$ is of complete continuous type and it generates a compact semigroup on $L^{1}(\Omega)$ (see Example 1.5.5, p. 19 and Lemma 2.7.2, p. 71 in Vrabie 
[25] and Theorem 1.7.9, p. 22 in Cârjă-Necula-Vrabie [11]). We rewrite (5.1) and (5.4) as an nonlinear multi-valued nonautonomous evolution systems.

Let us define the superposition operator $F: X \times X \rightarrow X$, by

$$
F(u, v)(x)=f(u(x), v(x))
$$

for each $(u, v) \in X \times X$ and a.e. for $x \in \Omega$, and the multi-function $G: L^{\infty}(\Omega) \times L^{\infty}(\Omega) \rightsquigarrow X$ by

$G(u, v)=\left\{g \in L^{\infty}(\Omega), \quad g_{1}(u(x), v(x)) \leq g(x) \leq g_{2}(u(x), v(x))\right.$ a.e. for $\left.x \in \Omega.\right\}$

Let us observe that $F$ is well-defined, continuous on $X \times X$ and is globally Lipschitz with respect to its second argument, i.e. $u$. Since $g_{1}, g_{2}$ satisfy $\left(\mathrm{H}_{4}\right)$, it follows that $G$ is strongly weakly u.s.c. with nonempty, convex, closed and bounded values in $L^{\infty}(\Omega)$. Then, $G$ has weakly compact values in $L^{1}(\Omega)$.

Let us define $\widetilde{F}: X \times X \rightarrow X$ and $\widetilde{G}: X \times X \rightarrow X$ by

$$
\widetilde{F}(u, v)(x)=\widetilde{f}(u(x), v(x)) \text { and } \widetilde{G}(u, v)(x)=\widetilde{g}(u(x), v(x))
$$

for each $(u, v) \in X \times X$ and a.e. for $x \in \Omega$. Since $\tilde{f}$ and $\widetilde{g}$ are continuous and have sublinear growth, $\widetilde{F}$ and $\widetilde{G}$ are well-defined, continuous and have sublinear growth. We rewrite (5.1) and (5.4) as a nonlinear system

$$
\left\{\begin{array}{l}
u^{\prime}(t)=A u(t)+F(u(t), v(t)) \\
v^{\prime}(t) \in B v(t)+G(u(t), v(t)) \\
u(\tau)=\xi, v(\tau)=\eta
\end{array}\right.
$$

and

$$
\left\{\begin{array}{l}
u^{\prime}(t)=A u(t)+\widetilde{F}(u(t), v(t)) \\
v^{\prime}(t)=B v(t)+\widetilde{G}(u(t), v(t)) \\
u(0)=u_{0}, v(0)=v_{0}
\end{array}\right.
$$

respectively.

We recall that if $\rho: \mathbb{R} \rightarrow \mathbb{R}$ is a nondecreasing and continuous function with $\rho(0)=0$, the $C^{0}$-solution $z \in C\left([0, T] ; L^{1}(\Omega)\right)$ of the problem

$$
\begin{cases}z_{t}=\Delta \rho(z)+h(t) & \text { in } Q_{0, T} \\ \rho(u)=0 & \text { on } \Sigma_{0, T} \\ z(0, x)=z_{0}(x) & \text { in } \Omega,\end{cases}
$$

satisfies

$$
\|z(t)\|_{L^{\infty}(\Omega)} \leq\left\|z_{0}\right\|_{L^{\infty}(\Omega)}+\int_{0}^{t}\|h(s)\|_{L^{\infty}(\Omega)} d s
$$

for each $t \in[0, T]$, whenever $z_{0} \in L^{\infty}(\Omega)$ and $h \in L^{\infty}\left(0, T ; L^{\infty}(\Omega)\right)$. See Example 1.1, p. 27 in Vrabie [24].

Let us prove now that $\mathcal{K}$ is viable with respect to $(A+F, B+G)$. Since $\xi \geq 0$ and $F(u, v) \geq 0$, in view of Lemma 13.5.1, p. 273 in Cârjă-NeculaVrabie [11], it follows that each solution $(u, v)$ of (5.1) satisfies $u \geq 0$.

Let $\widetilde{\mathcal{K}} \subseteq \mathbb{R}_{+} \times L^{\infty}(\Omega) \times L^{\infty}(\Omega)$ be defined by 


$$
\widetilde{\mathcal{K}}=\left\{(t, u, v) \in \mathbb{R}_{+} \times L^{\infty}(\Omega) \times L^{\infty}(\Omega) ;(t, u, v) \text { satisfies (5.11) below }\right\}
$$

$$
u(x) \leq \widetilde{u}(t, x), \quad v(x) \geq \widetilde{v}(t, x)
$$

a.e. for $x \in \Omega$. To prove that $\mathcal{K}$ is viable with respect to $(A+F, B+G)$ it suffices to show that $\widetilde{\mathcal{K}}$ is viable with respect to $(A+F, B+G)$. In view of Corollary 3.1 , we have merely to check the tangency condition

$$
(1, F(\xi, \eta), G(\xi, \eta)) \in \mathcal{T}_{\mathcal{S}_{\widetilde{\mathcal{K}}}^{\mathcal{A}}}^{\mathcal{A}}(\tau, \xi, \eta)
$$

for each $(\tau, \xi, \eta) \in \widetilde{\mathcal{K}}$, where $\mathcal{A}=(0, A, B)$. To this aim, it suffices to prove that, for each $(\tau, \xi, \eta) \in \widetilde{\mathcal{K}}$, we have

$$
\lim _{h \downarrow 0} \frac{1}{h} \operatorname{dist}((\tau+h, u(\tau+h, \tau, \xi, \eta, F(\xi, \eta)), v(\tau+h, \tau, \xi, \eta, G(\xi, \eta))) ; \widetilde{\mathcal{K}})=0,
$$

where

$$
v(\tau+\cdot, \tau, \xi, \eta, G(\xi, \eta))=\{v(\tau+\cdot, \tau, \xi, \eta, g) ; g \in G(\xi, \eta)\}
$$

and $(u(\cdot, \tau, \xi, \eta, f), v(\cdot, \tau, \xi, \eta, g))$ is the unique $C^{0}$-solution of the Cauchy problem

$$
\left\{\begin{array}{l}
u^{\prime}(t)=A u(t)+f \\
v^{\prime}(t)=B v(t)+g \\
u(\tau)=\xi, v(\tau)=\eta .
\end{array}\right.
$$

Let $(\tau, \xi, \eta) \in \widetilde{\mathcal{K}}$. To prove (5.13) it suffices to show that, for each $h>0$, there exists $\left(u_{h}, v_{h}\right) \in X \times X$ and $g_{h} \in G(\xi, \eta)$, with $\left(\tau+h, u_{h}, v_{h}\right) \in \widetilde{\mathcal{K}}$ and

$$
\left\{\begin{array}{l}
\lim _{h \downarrow 0} \frac{1}{h}\left\|u(\tau+h, \tau, \xi, \eta, F(\xi, \eta))-u_{h}\right\|_{L^{1}(\Omega)}=0 \\
\lim _{h \downarrow 0} \frac{1}{h}\left\|v\left(\tau+h, \tau, \xi, \eta, g_{h}\right)-v_{h}\right\|_{L^{1}(\Omega)}=0 .
\end{array}\right.
$$

Let us define $g_{h}(x)=g_{2}(\xi(x), \eta(x))$ a.e. for $x \in \Omega$ and $u_{h}$ and $v_{h}$ by

$$
\begin{aligned}
u_{h}= & u(\tau+h, \tau, \xi, \eta, F(\xi, \eta))+u(\tau+h, \tau, \widetilde{u}(\tau), \widetilde{v}(\tau), \widetilde{F}(\widetilde{u}(\cdot), \widetilde{v}(\cdot))) \\
& -u(\tau+h, \tau, \widetilde{u}(\tau), \widetilde{v}(\tau), \widetilde{F}(\widetilde{u}(\tau), \widetilde{v}(\tau)))
\end{aligned}
$$

and, respectively, by

$$
\begin{aligned}
v_{h}= & v\left(\tau+h, \tau, \xi, \eta, g_{h}\right)+v(\tau+h, \tau, \widetilde{u}(\tau), \widetilde{v}(\tau), \widetilde{G}(\widetilde{u}(\cdot), \widetilde{v}(\cdot))) \\
& -v(\tau+h, \tau, \widetilde{u}(\tau), \widetilde{v}(\tau), \widetilde{G}(\widetilde{u}(\tau), \widetilde{v}(\tau))) .
\end{aligned}
$$

Since $\xi \leq \widetilde{u}(\tau), \eta \geq \widetilde{v}(\tau)$ and $F(\xi, \eta) \leq \widetilde{F}(\xi, \eta)$, from the monotonicity properties of $\widetilde{f}$, we obtain $F(\xi, \eta) \leq \widetilde{F}(\xi, \eta) \leq \widetilde{F}(\widetilde{u}(\tau), \widetilde{v}(\tau))$ and then

$$
u_{h} \leq u(\tau+h, \tau, \widetilde{u}(\tau), \widetilde{v}(\tau), \widetilde{F}(\widetilde{u}(\cdot), \widetilde{v}(\cdot)))=\widetilde{u}(\tau+h) .
$$

Similarly, since $g_{2} \geq \widetilde{g}$, from the monotonicity properties of $\widetilde{g}$, we deduce

$$
g_{h} \geq \widetilde{G}(\xi, \eta) \geq \widetilde{G}(\widetilde{u}(\tau), \widetilde{v}(\tau)) .
$$


So, we get

$$
v_{h} \geq v(\tau+h, \tau, \widetilde{u}(\tau), \widetilde{v}(\tau), \widetilde{G}(\widetilde{u}(\cdot), \widetilde{v}(\cdot)))=\widetilde{v}(\tau+h) .
$$

Thus we have $\left(\tau+h, u_{h}, v_{h}\right) \in \widetilde{\mathcal{K}}$.

Using the continuity of $\widetilde{F}, \widetilde{G}, \widetilde{u}$ and $\widetilde{v}$, we obtain both

$$
\begin{aligned}
& \lim _{h \downarrow 0} \frac{1}{h}\left\|u(\tau+h, \tau, \xi, \eta, F(\xi, \eta))-u_{h}\right\|_{L^{1}(\Omega)} \\
& \quad \leq \lim _{h \downarrow 0} \frac{1}{h} \int_{\tau}^{\tau+h}\|\widetilde{F}(\widetilde{u}(s), \widetilde{v}(s))-\widetilde{F}(\widetilde{u}(\tau), \widetilde{v}(\tau))\|_{L^{1}(\Omega)} d s=0
\end{aligned}
$$

and

$$
\begin{aligned}
& \lim _{h \downarrow 0} \frac{1}{h}\left\|v\left(\tau+h, \tau, \xi, \eta, g_{h}\right)-v_{h}\right\|_{L^{1}(\Omega)} \\
& \quad \leq \lim _{h \downarrow 0} \frac{1}{h} \int_{\tau}^{\tau+h}\|\widetilde{G}(\widetilde{u}(s), \widetilde{v}(s))-\widetilde{G}(\widetilde{u}(\tau), \widetilde{v}(\tau))\|_{L^{1}(\Omega)} d s=0 .
\end{aligned}
$$

and so both relations in (5.15) are satisfied. This completes the proof of the viability of $\widetilde{\mathcal{K}}$ and consequently the viability of $\mathcal{K}$.

Let us observe that $\mathcal{K}$ satisfies the next property: for each sequence $\left(t_{n}, \xi_{n}, \eta_{n}\right)_{n}$ in $\mathcal{K}$ with $\lim _{n}\left(t_{n}, \xi_{n} \eta_{n}\right)=(t, \xi, \eta)$ and $t<T_{\mathcal{K}}$, where $T_{\mathcal{K}}$ is given by (5.16) below, it follows that $(t, \xi, \eta) \in \mathcal{K}$.

$$
T_{\mathcal{K}}=\sup \left\{t \in \mathbb{R} ; \text { there exist }(\xi, \eta) \in L^{\infty}(\Omega) \times L^{\infty}(\Omega) \text {, with }(t, \xi, \eta) \in \mathcal{K}\right\} .
$$

Since $F$ is bounded and $G$ has bounded values in $L^{\infty}(\Omega)$, it follows that each $C^{0}$-solution $(u, v):[\tau, T) \rightarrow X \times X$ of (5.1) with $(t, u(t), v(t)) \in \mathcal{K}$, can be continued to a global one, i.e., defined on $\left[\tau, T_{\mathcal{K}}\right)$ satisfying the very same condition on $\left[\tau, T_{\mathcal{K}}\right)$. Since $(\widetilde{u}, \widetilde{v})$ is defined on $\mathbb{R}_{+}$and $(t, \widetilde{u}(t), \widetilde{v}(t)) \in \mathcal{K}$ for each $t \in[0, \infty)$ we conclude that $T_{\mathcal{K}}=\infty$ and this completes the proof.

\section{Acknowledgments}

The author would like to express her warmest thanks to the anonymous referee for his/her critical comments and remarks who have contributed to a substantial improvement of the presentation.

\section{References}

[1] Ainseba, B., Aniţa, S.: Internal stabilization for reaction-diffusion problem modeling a predator-prey system. Nonlinear Anal. 61, 491-501 (2005)

[2] Aris, R.: The Mathematical Theory of Diffusion and Reaction in Permeable Catalysts. Oxford University Press (Clarendon), London (1975)

[3] Aubin, J.P.: Viability Theory. Birkhäuser, Boston (1991) 
[4] Barbu, V.: Nonlinear Semigroups and Differential Equation in Banach Spaces. Editura Academiei, Bucureşti, Noordhoff (1976)

[5] Bebernes, W., Schuur, I.D.: The Ważewski topological method for contingent equations. Ann. Math. Pure Appl. 87, 271-278 (1970)

[6] Bothe, D.: Nonlinear evolutions with Carathéodory forcing. J. Evol. Equ. 3, 375-394 (2003)

[7] Burlică, M.: Viability for semi-multi-valued reaction-diffusion systems. In: International Conference on Numerical Analysis and Applied Mathematics, AIP Conference Proceedings, vol. 1048, pp. 126-129 (2008)

[8] Burlică, M., Roşu, D.: A viability result for semilinear reaction-diffusion systems I. In: Proceedings of the International Conference on Applied Analysis and Differential Equations, pp. 31-44, Iaşi, România, 4-9 Sept 2006

[9] Burlică, M., Roşu, D.: A viability result for semilinear reaction-diffusion systems II. An. Ştiinţ. Univ. Al. I. Cuza Iaşi, Secţ. I a, Matematică 2, 361-382 (2008)

[10] Cârjă, O.: Some Methods of Nonlinear Functional Analysis. Matrix Rom, Bucureşti (2003) (Romanian)

[11] Cârjă, O., Necula, M., Vrabie, I.I.: Viability, invariance and applications. North Holland Math. Stud. 207, 350 (2007)

[12] Cârjă, O., Necula, M., Vrabie, I.I.: Necessary and sufficient conditions for viability for semilinear differential inclusions. Trans. Am. Math. Soc. 361, 343-390 (2009)

[13] Cârjă, O., Necula, M., Vrabie, I.I.: Necessary and sufficient conditions for viability for nonlinear evolution inclusions. Set Valued Anal. 16, 701-731 (2008)

[14] Deimling, K.: Multivalued differential equations on closed sets. Differ. Integr. Equ. 1, 23-30 (1988)

[15] Hukuhara, M.: Sur un théorème de Kneser. J. Fac. Sci. Univ. Tokyo Sect. I 6, 329-344 (1953)

[16] Lakshmikantham, V., Leela, S.: Nonlinear differential equations in abstract spaces. In: International Series in Nonlinear Mathematics, vol. 2, Pergamon Press, New York (1981)

[17] Murray, J.D.: Mathematical biology: I. An introduction. In: Interdisciplinary Applied Mathematics, 3rd edn, vol. 17, Springer, Berlin (2002)

[18] Nagumo, M.:Über die Lage der Integralkurven gewöhnlicher Differentialgleichungen. Proc. Phys. Math. Soc. Jpn. 24, 551-559 (1942)

[19] Necula, M., Vrabie, I.I.: A viability result for a class of fully nonlinear reactiondiffusion systems. Nonlinear Anal. 69, 1732-1743 (2008)

[20] Papageorgiou, N.S.: Viability theorems for nonautonomous differential inclusions with nonconvex domain. Math. Jpn. 40, 67-77 (1994) 
[21] Pavel, N.H.: Invariant sets for a class of semi-linear equations of evolution. Nonlinear Anal. 1, 187-196 (1977)

[22] Roşu, D.: Viability for nonlinear multi-valued reaction-diffusion systems. In: International Conference on Numerical Analysis and Applied Mathematics, AIP Conference Proceedings, vol. 1048, pp. 458-461 (2008)

[23] Shi, S.Z.: Viability theorems for a class of differential operator inclusions. J. Differ. Equ. 79, 232-257 (1989)

[24] Vrabie, I.I.: Compactness methods and flow-invariance for perturbed nonlinear semigroups. An. Ştiinţ. Univ. Al. I. Cuza Iaşi Secţ. I a, Matematică. 27, 117-125 (1981)

[25] Vrabie, I.I.: Compactness methods for nonlinear evolutions. In: Pitman Monographs and Survey in Pure and Applied Mathematics, 2nd edn, vol. 75, Longman, London (1995)

D. Roşu

Department of Mathematics,

Technical University "Gh.Asachi",

Bvd. Carol I., 700506 Iasi, Romania

e-mail: rosudaniela100@yahoo.com

Received: 25 February 2008.

Revised: 14 December 2009.

Accepted: 15 February 2010. 